\title{
Preheating in hybrid inflation
}

\author{
Juan García-Bellido \\ Theory Division, CERN, CH-1211 Geneva 23, Switzerland
}

Andrei Linde

Department of Physics, Stanford University, Stanford, California 94305-4060

(Received 20 November 1997; published 7 April 1998)

\begin{abstract}
We investigate the possibility of preheating in hybrid inflation. This scenario involves at least two scalar fields: the inflaton field $\phi$, and the symmetry breaking field $\sigma$. We found that the behavior of these fields after inflation, as well as the possibility of preheating (particle production due to parametric resonance), depends crucially on the ratio of the coupling constant $\lambda$ (self-interaction of the field $\sigma$ ) to the coupling constant $g^{2}$ (interaction of $\phi$ and $\sigma$ ). For $\lambda \gg g^{2}$, the oscillations of the field $\sigma$ soon after inflation become very small, and all the energy is concentrated in the oscillating field $\phi$. For $\lambda \sim g^{2}$ both fields $\sigma$ and $\phi$ oscillate in a rather chaotic way, but eventually their motion stabilizes, and parametric resonance with production of $\chi$ particles becomes possible. For $\lambda \ll g^{2}$ the oscillations of the field $\phi$ soon after inflation become very small, and all the energy is concentrated in the oscillating field $\sigma$. Preheating can be very efficient if the effective masses of the fields $\phi$ and $\sigma$ are much greater than the Hubble constant at the end of inflation, since those fields can then oscillate many times per $e$-fold, with a large amplitude. Preheating can also be efficient if these fields are coupled to other light scalar (or vector) fields $\chi$. In the recently proposed hybrid models with a second stage of inflation after the phase transition, both preheating and usual reheating are inefficient. Therefore for a very long time the universe remains in a state with vanishing pressure. As a result, density contrasts generated during the phase transition in these models can grow and collapse to form primordial black holes. Under certain conditions, most of the energy density after inflation will be stored in small black holes, which will later evaporate and reheat the universe. [S0556-2821(98)04510-X]
\end{abstract}

PACS number(s): $98.80 . \mathrm{Cq}$

\section{INTRODUCTION}

In most inflationary models the energy density at the end of inflation is released when the scalar field oscillates near the minimum of its potential, creates elementary particles, which later collide, decay into other particles, and eventually thermalize via various interactions [1]. The original version of the theory of reheating of the universe was based on the idea that the oscillating scalar field can be considered as a collection of scalar particles, each of which decays independently [2]. This simple picture, based on perturbation theory, is indeed valid in many inflationary models. However, recently it was shown that in many versions of chaotic inflation there is an additional stage when the oscillating scalar field produces other fields in a regime of parametric resonance. This regime was called "preheating" [3]. The process is nonperturbative and in certain cases it can be extremely efficient. It may change the final value of the temperature of the universe after reheating [3,4], it may lead to specific nonthermal phase transitions [5], and it may provide a new mechanism for the generation of the baryon asymmetry [6]. For a detailed discussion of the theory of preheating see $[7,8]$ and references therein.

In this paper we will discuss preheating in the context of hybrid inflation models [9]. These models became rather popular lately because they can be relatively easily formulated in the context of supersymmetric theories. They allow inflation to occur at very small values of scalar fields, and at a relatively small energy density, which removes some restrictions on inflationary models that previously seemed al- most unavoidable. This scenario is also interesting from the point of view of microwave background anisotropies and large scale structure because it provides natural models for tilted primordial spectra of density perturbations [10].

Hybrid inflation models describe at least two classical scalar fields, $\phi$ and $\sigma$. After the end of inflation both of these fields oscillate, and the energy of their oscillations can be transferred to the production of $\phi$ and $\sigma$ particles, or to the energy of other particles interacting with these fields.

The investigation of reheating in these models should consist of several steps. First of all, one should study the oscillations of the fields $\phi$ and $\sigma$ after inflation, neglecting particle production. Until now, this important question was only partially analyzed in some special situations. As we will show, the behavior of these two fields after inflation crucially depends on the relation between coupling constants in the theory. In some cases soon after inflation one of these fields rapidly relaxes near the minimum of the effective potential, whereas the other one continues to oscillate. Then all the energy after inflation is concentrated in this one field, and reheating occurs due to its decay. We will show how to find which of the two fields will play this role. In some other cases, the motion of these two fields becomes chaotic. Both of these fields oscillate simultaneously, and their energy transfers from one field to another many times during these oscillations. Without investigation of this issue one cannot achieve a proper understanding of reheating in hybrid inflation.

The second issue to study is the possibility of a parametric resonance which would transfer energy of the homogeneous 
oscillating classical fields $\phi$ and $\sigma$ to the energy of elementary particles $\phi$ and $\sigma$. As we will see, this process in general is possible, but typically it is not very efficient. Then one should analyze a possibility of a resonant decay of the fields $\phi$ and $\sigma$ to some other scalar or vector particles.

When this investigation is completed, one should study other channels of decay of the classical fields $\phi$ and $\sigma$, such as the usual (non-resonant) decay to their own quanta and into other particles in accordance to the elementary theory of reheating [2]. Indeed, as it was shown in Ref. [7], this is a separate process, which differs from the resonant decay in the limit when parametric resonance becomes inefficient.

Until now, investigation of reheating in hybrid inflation concentrated on the non-resonant decay of the fields $\phi$ and $\sigma$, see e.g. [11]. The results obtained by this method may be quite correct in some models where there is no parametric resonance, but if parametric resonance is possible, then the theory of reheating becomes completely different.

The purpose of this paper is to investigate this issue. Our paper is not going to be a complete study of preheating in hybrid inflation. We will restrict ourselves to the investigation of the first stages of preheating in hybrid inflation, which is sufficient to identify the models where preheating may occur. A complete investigation of preheating in hybrid inflation can be achieved using the analytical methods developed in [7], together with numerical lattice simulations along the lines of Refs. [12].

The main part of our paper will be devoted to the standard version of hybrid inflation with one stage of inflation. One may also consider inflationary models with two stages of inflation [13]. We will show that in such models both preheating and usual reheating are inefficient. However, under some conditions reheating in such models may be rather efficient, but it occurs in a rather unusual way. In a recent paper [14] we have shown that large density perturbations could be produced in such models at the time corresponding to the beginning of the second stage of inflation. In that case, large density contrasts re-enter the horizon during the radiation era and can collapse to form black holes. Radiation pressure may prevent the formation of primordial black holes, unless their density contrast is of order one. However, if the process of reheating after inflation is very inefficient, then pressure after inflation will be negligible, and nothing will prevent the formation of primordial black holes. If these black holes have a mass of order $10^{-5}-10^{-9} \mathrm{~g}$, they will evaporate very early and reheat the universe [14], producing all the entropy we observe today. This is a very interesting possibility which needs to be further explored.

\section{THE STAGE OF HYBRID INFLATION}

The simplest realization of chaotic hybrid inflation is provided by the potential [9]

$$
V(\phi, \sigma)=\frac{1}{4 \lambda}\left(M^{2}-\lambda \sigma^{2}\right)^{2}+\frac{1}{2} m^{2} \phi^{2}+\frac{1}{2} g^{2} \phi^{2} \sigma^{2} .
$$

The bare masses $m$ and $M$ of the scalar fields $\phi$ and $\sigma$ are "dressed" by their mutual interaction. At large values of the fields, their effective masses squared are both positive and the potential has the symmetry $\sigma \leftrightarrow-\sigma$. At small values of the field $\phi$, the potential has a maximum at $\phi=\sigma=0$ and a global minimum at $\phi=0, \sigma=\sigma_{0} \equiv M / \sqrt{\lambda}$, where the above symmetry is broken.

The complete equations of motion for the homogeneous fields are

$$
\begin{aligned}
& \ddot{\phi}+3 H \dot{\phi}=-\left(m^{2}+g^{2} \sigma^{2}\right) \phi, \\
& \ddot{\sigma}+3 H \dot{\sigma}=\left(M^{2}-g^{2} \phi^{2}-\lambda \sigma^{2}\right) \sigma,
\end{aligned}
$$

subject to the Friedmann constraint,

$$
H^{2}=\frac{8 \pi}{3 M_{\mathrm{P}}^{2}}\left[\frac{1}{2} \dot{\phi}^{2}+\frac{1}{2} \dot{\sigma}^{2}+V(\phi, \sigma)\right] .
$$

Motion starts at large $\phi$, where the effective mass of the $\sigma$ field is large and the field is sitting at the minimum of the potential at $\sigma=0$. As the field $\phi$ decreases, its quantum fluctuations produce an almost scale invariant but slightly tilted spectrum of density perturbations $[9,15,16]$.

During the slow-roll of the field $\phi$, the effective mass of the triggering field is $m_{\sigma}^{2}=g^{2} \phi^{2}-M^{2}$. When the field $\phi$ acquires the critical value $\phi_{c} \equiv M / g$, fluctuations of the massless $\sigma$ field trigger the symmetry breaking phase transition that ends inflation. If the bare mass $M$ of the $\sigma$ field is large compared with the rate of expansion $H$ of the universe, the transition will be instantaneous and inflation will end abruptly, as in the original hybrid inflation model $[9,17]$. If on the contrary the bare mass $M$ is of the order of $H$, then the transition will be very slow and there is a possibility of having yet a few more $e$-folds of inflation after the phase transition, see Refs. [13,14].

When $\sigma=0$ the inflaton potential becomes $V(\phi)$ $=M^{4} / 4 \lambda+m^{2} \phi^{2} / 2$. Since the scalar field $\phi$ is of the order of $\phi_{c}=M / g$, for $m^{2} \ll g^{2} M^{2} / \lambda$ the energy density is dominated by the vacuum energy,

$$
H_{0}^{2}=\frac{2 \pi}{3 \lambda} \frac{M^{4}}{M_{\mathrm{P}}^{2}},
$$

to very good accuracy [16]. At that time $\sigma=0$, and one can neglect $\ddot{\phi}$ in the equation of motion for the field $\phi$, so that

$$
3 H_{0} \dot{\phi}=-m^{2} \phi .
$$

It is then possible to integrate the evolution equation of $\phi$,

$$
\begin{gathered}
\phi(N)=\phi_{c} \exp (r N), \\
r \simeq \frac{m^{2}}{3 H_{0}^{2}},
\end{gathered}
$$

where $N=H_{0}\left(t_{c}-t\right)$ is the number of $e$-folds to the phase transition.

Quantum fluctuations of the inflaton field $\phi$ produce long wavelength metric perturbations, $\mathcal{R}=H \delta \phi / \dot{\phi}$, where $\delta \phi$ is the amplitude of the field fluctuation when it crosses outside the Hubble scale. These fluctuations give rise to a continuum spectrum of metric perturbations which can be computed exactly in the case of hybrid inflation [16] 


$$
\mathcal{P}_{\mathcal{R}}=\frac{C(r)^{2}}{r^{2}} \frac{g^{2} M^{2}}{6 \pi \lambda M_{\mathrm{P}}^{2}} e^{-2 r N},
$$

where $C(r)=\Gamma[3 / 2-r] / 2^{r} \Gamma[3 / 2]$, and the spectral tilt can be evaluated as

$$
n-1=\frac{d \ln \mathcal{P}_{\mathcal{R}}(k)}{d \ln k}=2 r .
$$

Note that the tilt is always greater than one in this model. Observations made on a wide range of scales, from Cosmic Background Explorer (COBE) Differential Microwave Radiometer (DMR) to CAT experiments, impose strong constraints on the amplitude and tilt of the primordial spectrum [18]

$$
\begin{aligned}
\mathcal{P}_{\mathcal{R}}^{1 / 2} & =5 \times 10^{-5}(0.99 \pm 0.06), \\
n & =0.91 \pm 0.10
\end{aligned}
$$

In the limit $m \ll H_{0}$, we have $r \simeq m^{2} / 3 H_{0}^{2}$, see Eq. (7), and $C(r) \simeq 1$. This means that the amplitude of the curvature perturbation spectrum should satisfy [16]

$$
\frac{g}{\lambda \sqrt{\lambda}} \frac{M^{5}}{m^{2} M_{\mathrm{P}}^{3}} \simeq 3.5 \times 10^{-5},
$$

while the tilt of the spectrum is bounded by [15]

$$
\frac{\lambda}{\pi} \frac{m^{2} M_{\mathrm{P}}^{2}}{M^{4}}<0.25 \text {. }
$$

\section{THE END OF INFLATION AND THE ONSET OF THE STAGE OF OSCILLATIONS}

As we already mentioned, in the simplest version of the hybrid inflation scenario described above inflation ends as soon as the scalar field $\phi$ decreases below $\phi_{c}=M / g$. It will be important for us to investigate this process in a more detailed way, because it prepares the stage for the process of reheating which we are going to study. In particular, we should understand whether or not the "waterfall" process of symmetry breaking from $\sigma=0$ to $|\sigma|=M / \sqrt{\lambda}$ can be considered as a rolling down of a homogeneous field $\sigma$.

According to the classical equations of motion, the field $\sigma=0$ cannot change its value because the first derivative of the effective potential at $\sigma=0$ vanishes. The process of spontaneous symmetry breaking in this case occurs due to the exponential growth of quantum fluctuations. Indeed, the field $\sigma$ has a (negative) effective mass squared $-\mu^{2}(\phi)$ $=g^{2}\left(\phi^{2}-\phi_{c}^{2}\right)$, which vanishes at the critical point, but becomes large and grows up to $\mu(0)=M$ as the field $\phi$ slides towards $\phi=0$. Quantum fluctuations of the scalar field $\sigma$ with momentum $\mathbf{k}$ grow as $\exp w_{k} t$, where $w_{k}=\sqrt{\mu^{2}-k^{2}}$ and $k=|\mathbf{k}|$. Symmetry breaking occurs due to the growth of fluctuations with $k<\mu$. This process produces an inhomogeneous distribution of the field $\sigma$ with $\langle\sigma\rangle=0$.

The resulting distribution of the scalar field $\sigma$ is relatively homogeneous on a scale $l \sim \mu^{-1}$ or even somewhat greater [19] because the rate of exponential growth is maximal at $k=0$, and the fluctuations with $k>\mu$ do not grow at all.
However, in our case we have an additional complication: the effective mass squared $-\mu^{2}(\phi)=g^{2}\left(\phi^{2}-\phi_{c}^{2}\right)$ changes in time. In order to estimate a typical scale on which the growth of the field $\sigma$ remains relatively homogeneous we will do the following. We will find $\mu^{2}$ as a function of time $\Delta t$ from the moment when $\phi=\phi_{c}$. The exponential growth becomes efficient when $\mu \sim \Delta t^{-1}$. Later, $\mu(t)$ continues growing, and all modes $\sigma_{k}$ which began growing at the moment $\Delta t$, will continue growing exponentially with approximately equal speed, and eventually trigger a more rapid motion of the field $\sigma$, see the next two sections. This motion will preserve initial homogeneity on a scale smaller than $\mu^{-1} \sim \Delta t$, which we are now going to determine.

The motion of the field $\sigma$ during this stage occurs due to the negative curvature of the effective potential,

$$
\begin{aligned}
\mu^{2} & =g^{2}\left(\phi_{c}^{2}-\phi^{2}\right) \approx 2 g^{2} \phi_{c}|\dot{\phi}| \Delta t=2 M^{2} m^{2} \Delta t / 3 H \\
& =\sqrt{2 \lambda / 3 \pi} m^{2} M_{\mathrm{P}} \Delta t .
\end{aligned}
$$

Perturbations of the field begin to grow exponentially at $\Delta t$ $\sim \mu^{-1}$, which gives

$$
\mu^{3} \sim \sqrt{\lambda} m^{2} M_{\mathrm{P}}
$$

Comparison of $\mu$ with $H \sim M^{2} / M_{\mathrm{P}} \sqrt{\lambda}$ shows that $\mu \gg H$ under the "waterfall condition", $\lambda m M_{\mathrm{P}}^{2} \gg M^{3}$ which should be satisfied in ordinary hybrid inflation [9]. This is violated, however, in hybrid models with two stages of inflation $[13,14]$.

This means that within the time $\mu^{-1} \ll H^{-1}$ the mass squared of the field $\sigma$ changes from being much greater than $H^{2}$ to being much smaller than $-H^{2}$. Therefore there will be no specific inflationary fluctuations of the field $\sigma$ with the wavelength $H^{-1}$, but instead we will have fluctuations of this field with the wavelength $\mu^{-1}$, which will give us the typical scale of homogeneity $l \sim \mu^{-1} \sim\left(m^{2} M_{\mathrm{P}} \sqrt{\lambda}\right)^{-1 / 3}$.

Now we should compare this length with other characteristic parameters which we will consider in our study of preheating. There are two orthogonal modes of oscillation near the minimum of the effective potential, which we will encounter in our discussion. The first mode corresponds to the oscillation of the field $\sigma$ at $\phi=0$; the corresponding frequency is given by $\bar{m}_{\sigma}=\sqrt{2} M$. One can easily verify that $\mu \ll M$ for $\lambda M_{\mathrm{P}}^{2} \gg m^{2}$, which is satisfied in all hybrid inflation models. The only possible exception known to us is the model of Refs. [13,14] with two different stages of inflation; we will discuss reheating in this model separately.

Another, orthogonal mode, corresponds to the frequency $\bar{m}_{\phi}=g M / \sqrt{\lambda}$, see the next section. One can show that $\mu$ $\ll \bar{m}_{\phi}$ if $m \ll 10^{-3} M_{\mathrm{P}} \lambda^{1 / 2} g^{-3 / 4}$. This condition is also satisfied in most versions of the hybrid inflation model.

The typical wavelength of perturbations produced by parametric resonance usually is not much different from the frequency of oscillations. Therefore our estimates indicate that in our investigation of preheating in the usual hybrid inflation one can treat the oscillating fields $\phi$ and $\sigma$ as homogeneous. 


\section{PREHEATING}

Here we are interested in the behavior of the fields after the end of inflation, as the fields oscillate around their minima, producing particles. The qualitative behavior is very different in two opposite limits, depending on the relation between the couplings $\lambda$ and $g^{2}$, and independently of the relation among the bare masses, $m \ll H<M$.

We will show that explosive preheating of $\phi$ and $\sigma$ particles is not very efficient in hybrid inflation. However, we will consider an extra scalar field $\chi$, coupled to both $\phi$ and $\sigma$,

$$
V(\chi)=\frac{1}{2} h_{1}^{2} \phi^{2} \chi^{2}+\frac{1}{2} h_{2}^{2} \sigma^{2} \chi^{2},
$$

which will allow explosive production of $\chi$ particles in hybrid inflation models for certain values of $h_{1}$ and $h_{2}$.

In order to study the production of particles of all fields we should analyze the equations of motion for the quantum fluctuations of the scalar fields. In linear perturbation theory we find

$$
\begin{gathered}
\ddot{\delta} \phi_{k}+3 H \dot{\delta} \phi_{k}+\left(\frac{k^{2}}{a^{2}}+m_{\phi}^{2}\right) \delta \phi_{k}=0, \\
\ddot{\delta} \sigma_{k}+3 H \dot{\delta} \sigma_{k}+\left(\frac{k^{2}}{a^{2}}+m_{\sigma}^{2}\right) \delta \sigma_{k}=0, \\
\ddot{\chi}_{k}+3 H \dot{\chi}_{k}+\left(\frac{k^{2}}{a^{2}}+m_{\chi}^{2}\right) \chi_{k}=0,
\end{gathered}
$$

where the effective masses are

$$
\begin{aligned}
& m_{\phi}^{2}=m^{2}+g^{2} \sigma^{2}+h_{1}^{2} \chi^{2}, \\
& m_{\sigma}^{2}=3 \lambda \sigma^{2}+g^{2} \phi^{2}-M^{2}+h_{2}^{2} \chi^{2}, \\
& m_{\chi}^{2}=h_{1}^{2} \phi^{2}+h_{2}^{2} \sigma^{2} .
\end{aligned}
$$

The rate of expansion of the universe is important only in the first few oscillations. However, it does play a role in ending the parametric resonance regime, since modes with physical momentum $k / a$ in a resonance band will be redshifted by the expansion and fall out of the band, shutting off particle production in that mode. It is possible to take into account the rate of expansion by redefining the fields $\varphi$ $=a^{3 / 2} \delta \phi, \psi=a^{3 / 2} \delta \sigma$ and $X=a^{3 / 2} \chi$. Since the scale factor soon goes like $a \propto t^{2 / 3}$, the equations of motion for these fields are the same as Eqs. (16)-(18) but without the friction term proportional to $3 H$. This will greatly simplify the analysis, while still taking into account the rate of expansion.

As we mentioned in the previous section, there are two fundamental frequencies of oscillations near the minimum of the effective potential: $\bar{m}_{\sigma}=\sqrt{2} M$ and $\bar{m}_{\phi}=g M / \sqrt{\lambda}$. Also, there are also two different scales of the fields $\phi$ and $\sigma: \phi_{c}$ $=M / g$, and $\sigma_{0}=M / \sqrt{\lambda}$.

If $\lambda \gg g^{2}$, one has $\sigma_{0} \ll \phi_{0}$ and $\bar{m}_{\sigma} \gg \bar{m}_{\phi}$. In this case oscillations of the field $\sigma$ tend to be insignificant. This field tends to follow adiabatically the position of the minimum of its instantaneous effective potential depending on the value of the slowly changing field $\phi$. Most of the energy of these two fields will be concentrated in the oscillations of the field $\phi$. Thus the theory of reheating in this regime should describe decay of the field $\phi$.

In the opposite limit, $\lambda \ll g^{2}$, one has $\sigma_{0} \gg \phi_{0}$ and $\bar{m}_{\sigma}$ $\ll \bar{m}_{\phi}$. In this case the situation is reversed. Oscillations of the field $\phi$ tend to be insignificant. This field tend to follow adiabatically the position of the minimum of its instantaneous effective potential depending on the value of the slowly changing field $\sigma$. Most of the energy of these two fields will be concentrated in the oscillations of the field $\sigma$, and the theory of reheating should describe decay of this field.

The situation $\lambda \sim g^{2}$ is more complicated. Both fields will oscillate with a comparable amplitude, transferring energy to each other in a rather chaotic way.

The constants $g$ and $\lambda$ could have in principle any value, as long as they satisfy the constraints (12) and (13) put together:

$$
\frac{g}{\sqrt{\lambda}} \frac{M}{M_{\mathrm{P}}}<5 \times 10^{-5} \text {. }
$$

For $M \sim 10^{-3} M_{\mathrm{P}}$, we have $g^{2} \ll \lambda$, like in the usual hybrid inflation [9], while for $M \sim 10^{-16} M_{\mathrm{P}}$, we can have $g^{2} \gg \lambda$, like in some hybrid models with two stages of inflation $[13,14]$. Finally there is a limiting case $g^{2} \sim \lambda$, which appears in the simplest models of hybrid inflation based on supergravity, see e.g. Refs. [17,20,21,11]. Reheating in these cases is completely different from each other. We will analyze the three different cases in the following sections.

\section{A. Case $g^{2} \ll \lambda$}

There are two well-differentiated regimes, depending on the ratio $\bar{m}_{\phi} / H=g M / \sqrt{\lambda} H$. For $\bar{m}_{\phi} / H \gg 1$, the amplitude of oscillations of the inflaton field $\phi$ after inflation is large, of order one, while for $\bar{m}_{\phi} / H \lesssim 1$, the amplitude could be very small. For both large and small ratios, the motion after the phase transition goes along the ellipse

$$
\lambda \sigma^{2}+g^{2} \phi^{2}=M^{2},
$$

in the plane $(\sigma, \phi)$, see Fig. 1. As the field $\sigma$ grows towards its minimum at $\sigma_{0}$, the field $\phi$ starts oscillating around zero, always along the ellipse. The main part of the oscillation comes from the $\phi$ field (perpendicular to the $\sigma$ direction), at $\sigma \simeq \sigma_{0}:$

$$
\frac{\phi}{\phi_{c}}=\Phi(t) \sin \bar{m}_{\phi} t .
$$

In the case of Fig. 1, Eq. (24) is a good approximation for the oscillations of the inflaton field, where $\Phi_{0} \simeq 1 / 15$ and $\Phi(t)$ $\propto 1 / t$, see Fig. 2. During the field's oscillations, the rate of expansion satisfies $H(t)=2 / 3 t$, which corresponds to a dustlike era, $a(t) \propto t^{2 / 3}$.

One should note that the details of the behavior of the fields $\phi$ and $\sigma$ may vary, depending on relations between different parameters of the theory. The results shown in Figs. 1 and 2 correspond to $\lambda=1, g=8 \times 10^{-4}, M=10^{-3} M_{\mathrm{P}}$ 


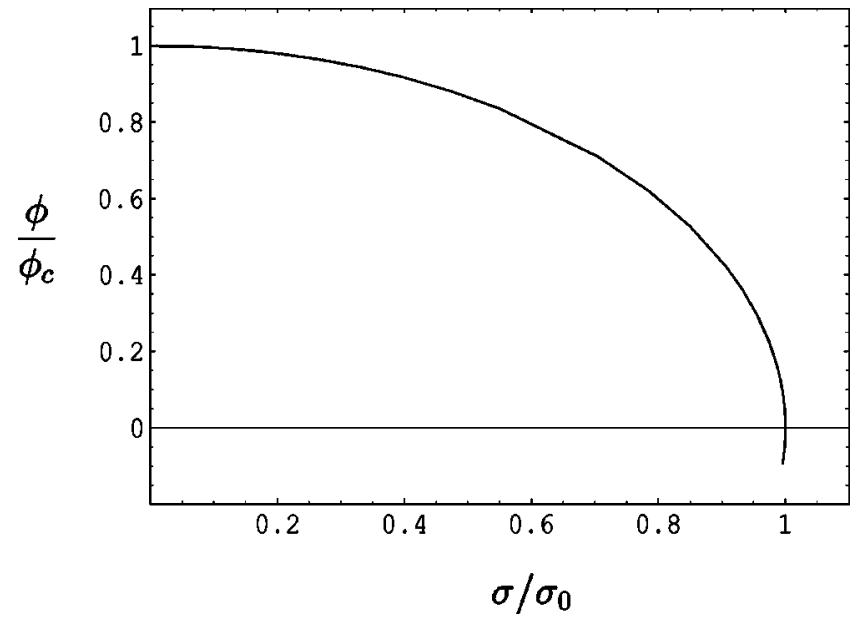

FIG. 1. The trajectory in field space $(\sigma, \phi)$ after the end of inflation, along the ellipse $\left(\phi / \phi_{c}\right)^{2}+\left(\sigma / \sigma_{0}\right)^{2}=1$. Oscillations occur around $\phi=0$ and $\sigma=\sigma_{0}$. This figure corresponds to $\lambda=1, g=8$ $\times 10^{-4}, M=10^{-3} M_{\mathrm{P}}, m=1.5 \times 10^{-7} M_{\mathrm{P}}$.

$\sim 10^{16} \mathrm{GeV}, m=1.5 \times 10^{-7} M_{\mathrm{P}} \sim 2 \times 10^{12} \mathrm{GeV}$. In this case $\bar{m}_{\phi}$ is of the same order as $H$ at the beginning of oscillations. This is one of the main reasons why the amplitude of oscillations is so small. Note that the Hubble constant at the beginning of oscillations is approximately ten times smaller than $H_{0}$. It is a very important circumstance, because it means that even before reheating begins, the energy density drops down by about two orders of magnitude. There are essentially no oscillations of the field $\sigma$, and the amplitude of oscillations of the field $\phi$ is relatively small.

However, one may consider models with different parameters, such that $\bar{m}_{\phi} \gg H$. For example, one may consider a model with $\lambda=1, \quad g=0.035, \quad M \sim 10^{12} \mathrm{GeV}$ and $m$ $=10^{3} \mathrm{GeV}$. In this case $\bar{m}_{\phi} \sim 10^{5} H$ and the figures look somewhat different: The amplitude of oscillations of the field $\sigma$ becomes noticeable, but still remains very small, whereas the initial amplitude of oscillations of the field $\phi$ becomes very large, comparable to $\phi_{c}$. This allows for a long stage of production of $\phi$ particles in the narrow resonance regime,

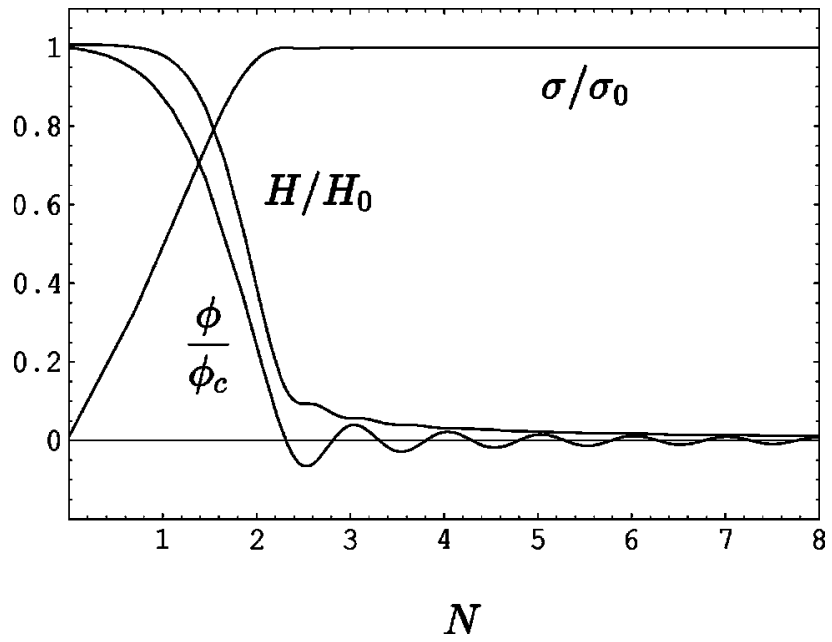

FIG. 2. The evolution after the end of inflation of $H / H_{0}, \phi / \phi_{c}$ and $\sigma / \sigma_{0}$, as a function of the number of oscillations of the field $\phi$, $N=\bar{m}_{\phi} t / 2 \pi$. before the back reaction and the expansion of the universe shifts the modes away from the resonance, see Ref. [7] and below.

It is possible to compute the production of particles of both fields during these oscillations. Let us start with the production of $\phi$-particles. The effective mass (19) of the field $\phi$ along the trajectory (23) becomes

$$
\begin{aligned}
m_{\phi}^{2} & =m^{2}+\frac{g^{2} M^{2}}{\lambda}\left(1-\frac{\phi^{2}}{\phi_{c}^{2}}\right)+h_{1}^{2} \chi^{2}, \\
& \simeq \bar{m}_{\phi}^{2}\left(1-\Phi^{2} \sin ^{2} \bar{m}_{\phi} t\right),
\end{aligned}
$$

where we have neglected $m^{2} \ll g^{2} M^{2} / \lambda$, see the discussion before Eq. (5), and defined $\bar{m}_{\phi} \equiv g M / \sqrt{\lambda}$. Initially $\left\langle\chi^{2}\right\rangle=0$, while the field $\phi$ oscillates around zero, see Eq. (24). Substituting into Eq. (16), we arrive at the Mathieu equation for the production of $\phi$ particles,

$$
\varphi_{k}^{\prime \prime}+\left[A_{\phi}(k)-2 q_{\phi} \cos 2 z\right] \varphi_{k}=0,
$$

where primes denote derivatives with respect to $z=\bar{m}_{\phi} t$ and

$$
\begin{aligned}
A_{\phi}(k) & =\frac{k^{2}}{a^{2} \bar{m}_{\phi}^{2}}+1+2 q_{\phi}, \\
q_{\phi} & =\frac{\Phi^{2}}{4} .
\end{aligned}
$$

To investigate parametric resonance which leads to the production of $\phi$ particles one should find exponentially growing solutions of the Mathieu equation (26) in an expanding universe. A detailed explanation of this approach to preheating can be found in Ref. [7]; we will not repeat it here. We will only recall that preheating can be especially efficient in the regime when $A_{\phi}(k) \lesssim 2 q_{\phi}$ and $q_{\phi} \gtrsim 1 / 4$, which corresponds to a broad parametric resonance. The resonance appears not for all $k$, but only for those $k$ belonging to the instability bands of the Mathieu equation. The concept of stability/instability bands is very useful if one can neglect the role of expansion of the universe; in a more general case one should solve Eq. (26) numerically and find out the modes which will grow specially fast. These modes will dominate particle production.

For a complete investigation of preheating one also needs to investigate those effects related to back reaction of produced particles and their rescattering $[7,8,12]$. We will make some comments on this issue later, but we will mainly concentrate on the first stages of preheating, where the corresponding effects can be neglected. This approach will allow us to identify those versions of hybrid inflation where preheating may be efficient.

For the model described by Figs. 1, 2 (with $\lambda=1, g=8$ $\left.\times 10^{-4}, M=10^{-3} M_{\mathrm{P}}, m=1.5 \times 10^{-7} M_{\mathrm{P}}\right)$ the initial value of $q$ is extremely small, $q_{\phi}=\Phi_{0}^{2} / 4 \simeq 10^{-3} \ll 1$. For $k \simeq 0$, we are in the narrow region of the first resonance band, $A=1$. In this case there are solutions of Eq. (26) that correspond to exponential instabilities with occupation numbers of quantum fluctuations given by $n_{k}(t) \propto \exp \left(2 \mu_{k} z\right)$, which can be interpreted as particle production [3]. However, the effective 
parameter $2 \mu_{k} \sim q$ will be extremely small and particle production will not be very efficient.

It is possible to evaluate the time it takes for a particular mode $k$ to cross out of the resonance band, $\Delta t \sim \mu_{k} H^{-1}$, see Ref. [3]. The occupation number then becomes $n_{k}$ $\sim \exp \left(2 \mu_{k}^{2} \bar{m}_{\phi} / H\right)$. Explosive production shuts down when $\Phi^{4}<32 H / \bar{m}_{\phi}$. In our model, this is true from the very beginning, and so we are confident that very little production of $\phi$-particles will occur.

In the models where the initial amplitude of the field $\phi$ is large (of the same order as $\phi_{c}$ ), production of $\phi$ particles can be much more efficient. For example, in the model with $\lambda$ $=1, g=0.035, M \sim 10^{12} \mathrm{GeV}$ and $m=10^{3} \mathrm{GeV}$ one has case $\bar{m}_{\phi} \sim 10^{5} \mathrm{H}$, which means that the field can make about $10^{4}$ oscillations before expansion of the universe becomes important. The initial value of the parameter $q$ is about $1 / 4$, see Eq. (28). This corresponds to the narrow resonance regime in the second instability band of the Mathieu equation.

The resonance in the second instability band at small $q$ typically is very narrow and inefficient. Moreover, one should check carefully whether this band actually exists, because Eq. (26) is correct only when one neglects oscillations of the field $\sigma$. Meanwhile these oscillations are not entirely negligible, so they may affect the narrow resonance in the second instability band. Indeed in our numerical investigation of the resonance in the coupled system of the fields $\phi$ and $\sigma$ we did not find any evidence of $\phi$ particle production. Even if this process happens, it is not really reheating; parametric resonance breaks the coherently oscillating field $\phi$ into a collection of $\phi$ particles. A subsequent perturbative decay of $\phi$ particles is necessary.

Let us consider now the production of $\sigma$-particles. The effective mass (20) along the trajectory (23) becomes

$$
\begin{aligned}
m_{\sigma}^{2} & =2 M^{2}\left(1-\frac{\phi^{2}}{\phi_{c}^{2}}\right)+h_{2}^{2} \chi^{2}, \\
& \simeq \bar{m}_{\sigma}^{2}\left(1-\Phi^{2} \sin ^{2} \bar{m}_{\phi} t\right),
\end{aligned}
$$

where initially $\left\langle\chi^{2}\right\rangle=0$ and $\bar{m}_{\sigma} \equiv \sqrt{2} M$. Substituting back into Eq. (17), we arrive at the Mathieu equation for the production of $\sigma$ particles,

$$
\psi_{k}^{\prime \prime}+\left[A_{\sigma}(k)-2 q_{\sigma} \cos 2 z\right] \psi_{k}=0,
$$

where $z=\bar{m}_{\phi} t$ and

$$
\begin{gathered}
A_{\sigma}(k)=\frac{k^{2}}{a^{2} \bar{m}_{\phi}^{2}}+\frac{\bar{m}_{\sigma}^{2}}{\bar{m}_{\phi}^{2}}+2 q_{\sigma}, \\
q_{\sigma}=\frac{\bar{m}_{\sigma}^{2}}{\bar{m}_{\phi}^{2}} \frac{\Phi^{2}}{4} .
\end{gathered}
$$

The ratio $\bar{m}_{\sigma}^{2} / \bar{m}_{\phi}^{2}=2 \lambda / g^{2}$, while $q \simeq\left(\lambda / g^{2}\right) \Phi^{2} / 2$. Since $\Phi$ $\ll 2 \sqrt{2 \lambda} / g$ in our case, we have that $A-2 q \gg \sqrt{q}$, i.e. far above the resonance band, and therefore we expect no explosive production of $\sigma$-particles from the oscillations of the field $\phi$.
Moreover, $\sigma$ particles can neither be produced at the next (perturbative) stage of the decay of the field $\phi$. Indeed, the effective mass of $\phi$-particles, $\bar{m}_{\phi} \sim g M / \sqrt{\lambda}$, in the case $g^{2}$ $\ll \lambda$, is much smaller than the mass of the $\sigma$ particles, $\sqrt{2} M$, so the decay of $\phi$ particles is kinematically impossible. Thus, in this regime the field $\phi$ cannot transfer its energy to $\sigma$ particles.

It is possible, however, to parametrically amplify fluctuations of another scalar (or vector) field $\chi$, which interacts with the fields $\phi$ and $\sigma$ in accordance with Eq. (15). Since oscillations will occur around $\phi=0$, while $\sigma \simeq \sigma_{0}$, in order to have particle production in this field we require that the induced mass from the symmetry breaking field $\sigma$ be much smaller than the corresponding oscillations from the inflaton field $\phi, h_{1}^{2} \phi^{2} \gg h_{2}^{2} \sigma_{0}^{2}$, which at the beginning of preheating corresponds to

$$
\lambda h_{1}^{2} \gg g^{2} h_{2}^{2} .
$$

It is always possible to find couplings $h_{1}, h_{2}$ that satisfy this constraint. Eventually, when the amplitude of oscillations of the $\phi$ field becomes

$$
\Phi(t)<\frac{h_{2} g}{h_{1} \sqrt{\lambda}},
$$

the explosive production of $\chi$-particles will end. This may happen before or after backreaction sets in, depending on the parameters of the model.

The corresponding Mathieu equation for the production of $\chi$-particles is given by

$$
X_{k}^{\prime \prime}+[A(k)-2 q \cos 2 z] X_{k}=0,
$$

where

$$
\begin{gathered}
A(k)=\frac{k^{2}}{a^{2} \bar{m}_{\phi}^{2}}+2 q, \\
q=\frac{h_{1}^{2} M^{2}}{g^{2} \bar{m}_{\phi}^{2}} \frac{\Phi^{2}}{4} .
\end{gathered}
$$

Initially, $q_{0}=\left(\lambda h_{1}^{2} / g^{4}\right) \Phi_{0}^{2} / 4 \simeq\left(\lambda h_{1}^{2} / g^{4}\right) 10^{-3}$, where we have assumed $h_{2}$ satisfies Eq. (33) and can be neglected. Since $\lambda \gg g^{2}$, it is possible to find natural values of the parameter $h_{1}$ that ensure $q_{0} \gg 1$. We are therefore in the broad parametric resonance region and explosive production of $\chi$ particles will occur. In this case there are solutions of Eq. (35) that correspond to exponential instabilities with occupation numbers of quantum fluctuations given by

$$
\begin{aligned}
n_{k}(t) & =\frac{\omega_{k}}{2}\left(\frac{\left|\dot{X}_{k}\right|^{2}}{\omega_{k}^{2}}+\left|X_{k}\right| 2\right)-\frac{1}{2} \\
& \simeq \frac{1}{2} \exp \left(2 \mu_{k} z\right),
\end{aligned}
$$

which can be interpreted as particle production [3], where the frequency $\omega_{k}$ is given by 


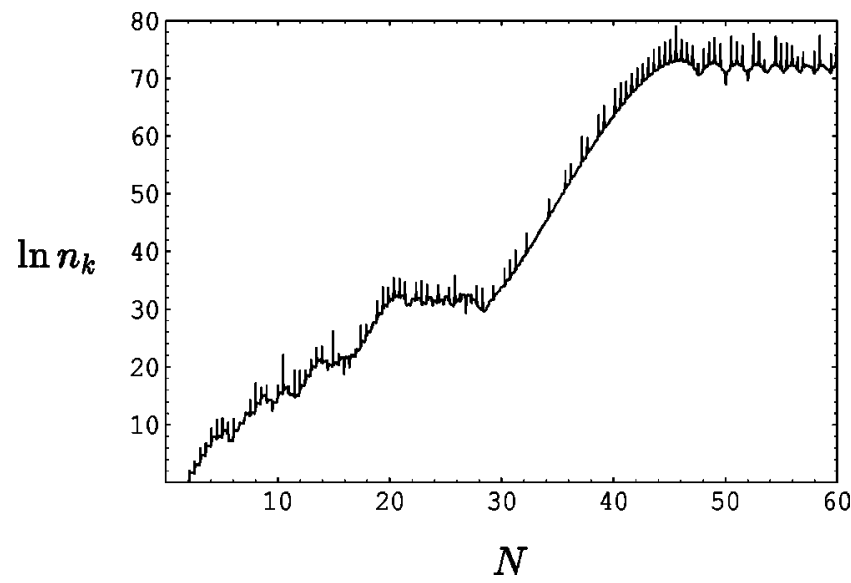

FIG. 3. The exponential growth of the occupation number $n_{k}$ of $\chi$-particles, for $k=\bar{m}_{\phi}$, as a function of the number of oscillations of the field $\phi, N=\bar{m}_{\phi} t / 2 \pi$. One can distinguish here the broad stochastic resonance regime. The typical value of the growth parameter is $\mu \simeq 0.13$ during the last stages of preheating.

$$
\omega_{k}^{2}=\frac{k^{2}}{a^{2}}+h_{1}^{2} \phi^{2} .
$$

We have shown the exponential growth of the occupation number $n_{k}$ of $\chi$-particles in Fig. 3, which determines the effective growth parameter $\mu_{k}$ in the broad resonance region, see Eq. (38). The model parameters used are $\lambda=1, h_{1}=g$ $=8 \times 10^{-4}, M=10^{-3} M_{\mathrm{P}}, m=1.5 \times 10^{-7} M_{\mathrm{P}}$, for the typical momentum $k=\bar{m}_{\phi}$. In this case, the typical growth parameter is relatively large, $\mu \simeq 0.13$, and particle production will be very efficient. Furthermore, for a large range of couplings we will enter the region of stochastic resonance, see Ref. [7].

The present case is analogous to the case found in [7] for a massive inflaton field, where we substitute $m \rightarrow \bar{m}_{\phi}$ $=g M / \sqrt{\lambda}$ and $g \rightarrow h_{1}$. All features found in that case will be present here, for a certain range of parameters. For instance, back reaction will set in at the time $t_{1}$ given by [7]

$$
N_{1}=\frac{\bar{m}_{\phi} t_{1}}{2 \pi}=\frac{5}{8 \pi \mu} \ln \frac{15}{h_{1}} \simeq 15
$$

when the expectation value of created particles satisfies

$$
\left\langle\chi^{2}\right\rangle_{1} \simeq \frac{\bar{m}_{\phi}^{2}}{h_{1}^{2}}=\frac{g^{2} M^{2}}{\lambda h_{1}^{2}}
$$

and the oscillating field $\phi$ acquires a large effective mass. At that time the parameter $q$ becomes

$$
q_{1}^{1 / 2}=\frac{q_{0}^{1 / 2}}{4 N_{1}} \simeq \frac{h_{1} \sqrt{\lambda}}{4 g^{2}} 10^{-4} .
$$

The efficiency of preheating at this stage is determined by the fraction of energy density in kinetic energy of $\chi$ particles, $\left\langle(\nabla \chi)^{2}\right\rangle_{1}=\bar{m}_{\phi}^{2} \phi_{c}^{2} \Phi_{1}^{2} q_{1}^{-1 / 2}$, see Ref. [7], which could be very small compared with the energy density remaining in the oscillations of the inflaton field, if $q_{1} \gg 1$.
Even after back reaction there is still production of $\chi$ particles in the broad resonance regime until the parameter $q$ falls below 1/4, see Ref. [7]. At that time,

$$
\left\langle\chi^{2}\right\rangle_{2} \simeq \phi_{c}^{2} \Phi^{2}\left(t_{2}\right)=\frac{4 g^{2} M^{2}}{\lambda h_{1}^{2}} q_{1}^{1 / 2},
$$

where we have used the relation $\Phi_{2} \simeq \Phi_{1} q_{1}^{-1 / 4}$ between the amplitude of oscillations at both times, see [7]. At this time the energy density is equally distributed between the kinetic energy of the $\chi$ particles and their interaction energy with the field $\phi$. In that case, $\left\langle(\nabla \chi)^{2}\right\rangle_{2}=\bar{m}_{\phi}^{2} \phi_{c}^{2} \Phi_{2}^{2} q_{1}^{1 / 2}=\bar{m}_{\phi}^{2} \phi_{c}^{2} \Phi_{1}^{2}$, see Ref. [7]. This result gets modified when including rescattering of $\chi$ particles with particles of the field $\phi$. The end of preheating occurs slightly earlier, when $\Phi_{r} \simeq 2.5 \Phi_{1} q_{1}^{-1 / 4}$, and the energy density is predominantly in the interaction energy between $\chi$ particles and the field $\phi, \rho_{\chi}$ $\sim 10^{-2} g^{2} \phi_{c}^{4} \Phi_{r}^{4}$.

A relevant question in the presence of preheating is whether non-thermal effects due to large occupation numbers of particles can restore the symmetry in the $\sigma$ field, see Ref. [7]. For this to occur, we need that the effective mass in the false vacuum be positive at the end of preheating,

$$
\begin{aligned}
V_{\sigma}^{\prime \prime}(0) & =-M^{2}+h_{2}^{2}\left\langle\chi^{2}\right\rangle_{2} \\
& =M^{2}\left(-1+\frac{4 g^{2} h_{2}^{2}}{\lambda h_{1}^{2}} q_{1}^{1 / 2}\right)>0 .
\end{aligned}
$$

We see that the condition for symmetry restoration in this model requires

$$
q_{1}^{1 / 2}=\frac{q_{0}^{1 / 2}}{4 N_{1}}>\frac{\lambda h_{1}^{2}}{4 g^{2} h_{2}^{2}} \gg 1,
$$

see Eq. (33). Unless the initial value $q_{0}$ is very large indeed, it is very difficult to satisfy this condition. There is however no fundamental reason why this should not happen, and, as a consequence, non-thermal symmetry restoration could in principle be possible in this model for certain relations between coupling constants. If this happens, the description of preheating given above will need to be modified.

Let us now study the opposite limit, $\lambda \ll g^{2}$, and see whether there is parametric resonance production of particles in that case.

\section{B. Case $\lambda \ll g^{2}$}

For $\lambda \ll g^{2}$, the most important mode of oscillations is the oscillation of the field $\sigma$ near the minimum of the effective potential at $\sigma_{0}=M / \sqrt{\lambda}$. The initial stages of oscillations can be very complicated. Depending on the relations between the parameters of the theory, the first motion of the fields $(\phi, \sigma)$ may occur either in the $\sigma$-direction, as in the standard hybrid inflation scenario [9], or in $\phi$ direction, as in Refs. [13,14]. However, in both cases the oscillations of the field $\phi$ eventually become small, and all energy becomes concentrated in the oscillations of the field $\sigma$, see Fig. 4.

Thus, just as in the case $\lambda \gg g^{2}$, the motion of the fields eventually becomes essentially one-dimensional, but now it 


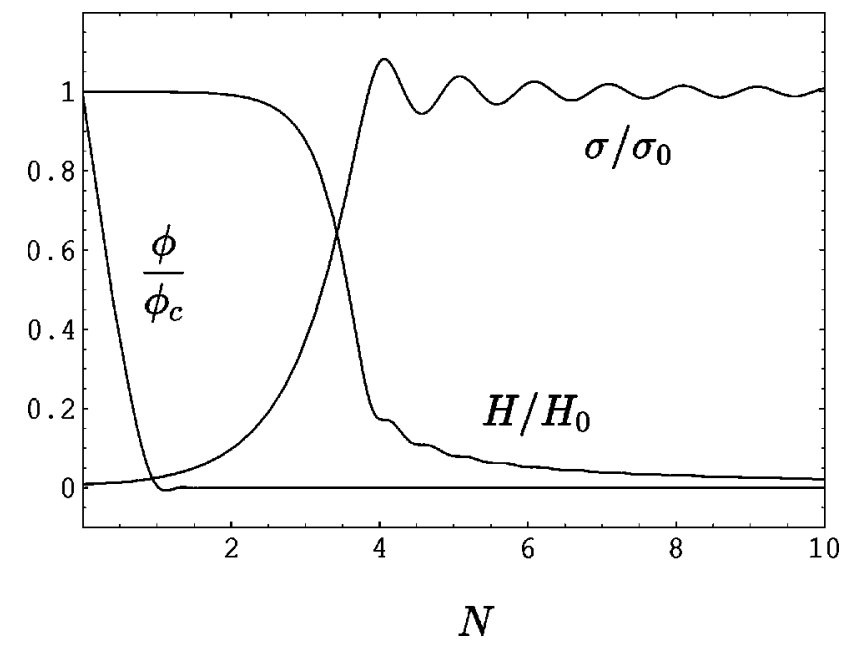

FIG. 4. The evolution after the end of inflation of $H / H_{0}, \phi / \phi_{c}$ and $\sigma / \sigma_{0}$, as a function of the number of oscillations of the field $\sigma$, $N=\bar{m}_{\sigma} t / 2 \pi$. This figure corresponds to $\lambda=2 \times 10^{-16}, g=1.4$ $\times 10^{-5}, M=m=10^{-8} M_{\mathrm{P}}$.

occurs in the $\sigma$ direction. The corresponding process is very similar to the one which occurs in new inflation. The oscillations of the field $\sigma$ can be represented in the following way:

$$
\frac{\sigma}{\sigma_{0}}=1+\Sigma(t) \sin \bar{m}_{\sigma} t
$$

This is a good approximation for the oscillations of the $\sigma$ field with a small amplitude $\Sigma_{0} \ll 1, \Sigma(t) \propto 1 / t$, see Fig. 4 .

It is then possible to compute the production of particles of both fields during the $\sigma$-oscillations. Let us start with the production of $\sigma$-particles. Before the symmetry breaking field starts oscillating, the field $\phi$ has negligible amplitude, so we will assume $\phi \simeq 0$. The effective mass (20) of the field $\sigma$ then becomes

$$
\begin{aligned}
m_{\sigma}^{2} & =2 M^{2}+3 \lambda\left(\sigma^{2}-\sigma_{0}^{2}\right)+h_{2}^{2}\left\langle\chi^{2}\right\rangle, \\
& \simeq \bar{m}_{\sigma}^{2}\left(1+3 \Sigma \sin \bar{m}_{\sigma} t\right),
\end{aligned}
$$

where $\left\langle\chi^{2}\right\rangle=0$ initially, and $\bar{m}_{\sigma}^{2} \equiv 2 M^{2}$. Substituting back into Eq. (17), we arrive at the Mathieu equation for the production of $\sigma$ particles,

$$
\psi_{k}^{\prime \prime}+\left[A_{\sigma}(k)+2 q_{\sigma} \sin 2 z\right] \psi_{k}=0
$$

where $z=\bar{m}_{\sigma} t / 2$ and ${ }^{1}$

$$
\begin{aligned}
A_{\sigma}(k) & =\frac{2 k^{2}}{a^{2} M^{2}}+4, \\
q_{\sigma} & =6 \Sigma .
\end{aligned}
$$

Initially in our model we have $\Sigma \simeq 1 / 12,\left\langle\chi^{2}\right\rangle=0$, so $q_{\sigma}$ $=6 \Sigma \simeq 0.5$, see Fig. 4. For $k \simeq 0$, we are in the narrow region

\footnotetext{
${ }^{1}$ We have neglected the $\Sigma^{2}$ and the $\Phi^{2}$ terms, which would have contributed as higher harmonics and soon be completely irrelevant.
}

of the second resonance band, $A=4$. There will be some production of $\sigma$-particles but very soon the modes will redshift away and production will shut down.

The effective mass of $\phi$ particles (19) for small $\phi$ and $m \ll \bar{m}_{\phi}$ becomes

$$
\begin{aligned}
m_{\phi}^{2} & =m^{2}+\frac{g^{2} M^{2}}{\lambda}+g^{2}\left(\sigma^{2}-\sigma_{0}^{2}\right)+h_{1}^{2}\left\langle\chi^{2}\right\rangle \\
& \simeq \bar{m}_{\phi}^{2}\left(1+2 \Sigma \sin \bar{m}_{\sigma} t\right),
\end{aligned}
$$

where $\left\langle\chi^{2}\right\rangle=0$ initially, and $\bar{m}_{\phi}=g M / \sqrt{\lambda}$. This corresponds to the Mathieu parameters

$$
\begin{aligned}
A_{\phi}(k) & =\frac{2 k^{2}}{a^{2} M^{2}}+\frac{2 g^{2}}{\lambda}, \\
q_{\phi} & =\frac{2 g^{2}}{\lambda} \Sigma .
\end{aligned}
$$

Therefore, for $\Sigma \ll 1$ one has $q_{\phi} \ll A_{\phi}(k)$. In this regime the resonance is very narrow, and one does not expect a large production of $\phi$ particles.

This conclusion remains valid for the perturbative decay of the oscillating scalar field $\sigma$ as well. Indeed, the effective mass of the field $\phi$ particles $\bar{m}_{\phi} \sim g M / \sqrt{\lambda}$ in the case $g^{2}$ $\gg \lambda$ is much greater than the mass of the $\sigma$ particles $\sqrt{2} M$, so the decay of $\sigma$ particles to $\phi$ particles is kinematically impossible. Thus, in this regime the field $\phi$ cannot transfer its energy to $\sigma$ particles. This is very similar to what happens in the case $g^{2} \ll \lambda$, where the energy is concentrated in the oscillating field $\phi$, which (for $g^{2} \ll \lambda$ ) cannot decay to $\sigma$ particles, see the previous section.

Parametric resonance with production of $\chi$ particles in principle could be possible. In the case where masses of particles near the minimum of the effective potential are not much greater than $H$, one can ignore the small $\phi$ oscillations. Then the $\chi$ particle production is described by the Mathieu equation

$$
\ddot{X}_{k}+\left[\frac{k^{2}}{a^{2}}+h_{2}^{2} \sigma_{0}^{2}+2 h_{2}^{2} \sigma_{0}^{2} \Sigma(t) \sin \bar{m}_{\sigma} t\right] X_{k}=0,
$$

see [7]. A change of variables $\bar{m}_{\sigma} t=2 z-\pi / 2$ reduces Eq. (55) to the Mathieu equation

$$
X_{k}^{\prime \prime}+[A(k)-2 q \cos 2 z] X_{k}=0 .
$$

Here $A(k)=2 k^{2} / a^{2} M^{2}+2 h_{2}^{2} / \lambda, \quad q=\left(2 h_{2}^{2} / \lambda\right) \Sigma, \quad z=\bar{m}_{\sigma} t / 2$, and primes denote differentiation with respect to $z$.

Note that $q<A(k) \Sigma$. In this case the Mathieu plot of stability/instability bands shows that as soon as the amplitude of oscillations of the field $\sigma$ becomes much smaller than $\sigma_{0}$, one has $\Sigma \ll 1$, which implies that $q \ll A(k)$. In this regime the resonance is narrow, and preheating is not very efficient [3]. In our case $\Sigma \ll 1$ from the very beginning of the period of oscillations, and $\chi$ particle production is inefficient. 
It is possible in principle to conceive a scalar field $\chi$ which couples not to $\sigma$ but to the difference $\sigma-\sigma_{0}$,

$$
m_{\chi}^{2}=h_{2}^{2}\left(\sigma-\sigma_{0}\right)^{2} .
$$

In such models production of $\chi$-particle can be very efficient. Let us analyze this case is more detail here.

Substituting Eq. (57) into Eq. (18), we arrive at the Mathieu equation for the production of $\chi$ particles, Eq. (35), with $z=\bar{m}_{\sigma} t$ and

$$
\begin{gathered}
A(k)=\frac{k^{2}}{a^{2} \bar{m}_{\sigma}^{2}}+2 q, \\
q=\frac{h_{2}^{2} M^{2}}{\lambda \bar{m}_{\sigma}^{2}} \frac{\Sigma^{2}}{4} .
\end{gathered}
$$

Initially, $\left\langle\chi^{2}\right\rangle=0$, so $q_{0}=\left(h_{2}^{2} / \lambda\right)\left(\Sigma^{2} / 8\right) \simeq\left(h_{2}^{2} / \lambda\right) 10^{-3}$, see Fig. 4. Since $\lambda \ll g^{2}$ in this case, we can easily get $q_{0} \gg 1$. This ensures explosive $\chi$-particle production with a large effective growth parameter $\mu_{k}$. As an example, we considered a model with $\lambda=2 \times 10^{-16}, h=10 g=1.4 \times 10^{-5}, M=m$ $=10^{-8} M_{\mathrm{P}}$. We have found that the typical growth parameter is rather large, $\mu \simeq 0.13$, and particle production is very efficient.

Note however that it is rather difficult (though not impossible) to invent a realistic model where $\chi$ particles have masses given by Eq. (57). We have found that it is possible in the context of supersymmetric models, but it requires explicit fine tuning of the superpotential of the theory. Thus, one may conclude that in a general class of hybrid inflation models where $\chi$ particles acquire a mass $h_{2} \sigma_{0}$ after spontaneous symmetry breaking, preheating for $\lambda \ll g^{2}$ is inefficient. This question should be reexamined in the theories with flat directions, and in the theories where $\chi$ particles have small mass due to an accidental cancellation of two large contribution. Such a situation may not be entirely unrealistic; remember that the Higgs doublet of the standard model has an extremely small mass as compared to the GUT scale.

In the version of the hybrid inflation scenario which we discussed until now the amplitude of oscillations of the field $\sigma$ was relatively small. However, our conclusions concerning parametric resonance for $\lambda \ll g^{2}$ can change considerably if one finds the models with $M \gg H$, which induce a quick end of inflation and a large amplitude of oscillations of the field $\sigma$ after inflation. For example, one may consider a model with $g=1, \lambda=10^{-2}, \quad m=10^{3} \mathrm{GeV}, \quad M=1.3 \times 10^{11} \mathrm{GeV}$. This gives $H \sim 2 \times 10^{4} \mathrm{GeV}, \bar{m}_{\phi}=5 \times 10^{7} H$ and $\bar{m}_{\sigma}=8$ $\times 10^{6} \mathrm{H}$. In such a model the amplitude of the oscillations of the field $\sigma$ is very large, $\Sigma \sim 1$, see Fig. 5. Moreover, this amplitude will not be damped by the expansion of the universe during $10^{6}$ oscillations of this field. As a result, in this model there will be a very efficient production of $\sigma, \phi$ and $\chi$ particles in a broad resonance regime, see Figs. 6 and 7.

These figures illustrate the growth of fluctuation neglecting back reaction. In fact, preheating in this model is so efficient that it completes within few oscillations. Indeed, let us estimate how strong should be the growth of $n_{k}$ in order to

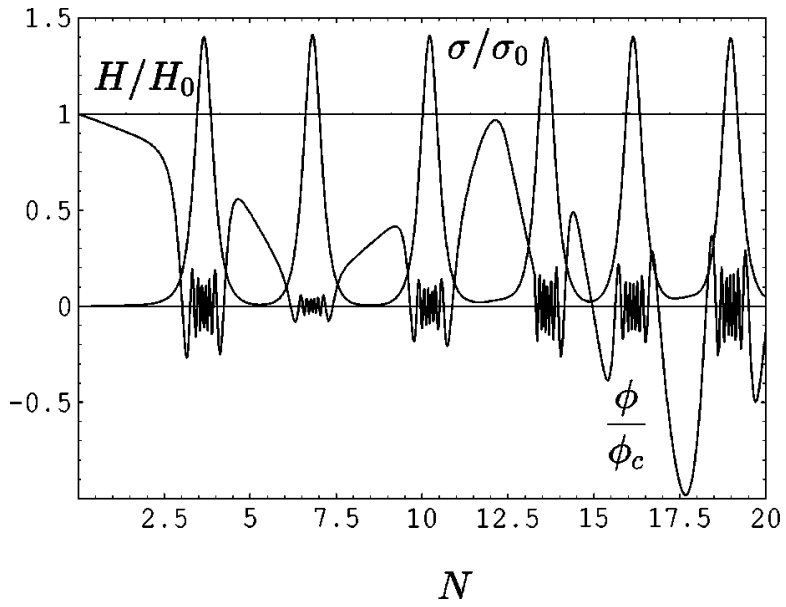

FIG. 5. The evolution after the end of inflation of $H / H_{0}, \phi / \phi_{c}$ and $\sigma / \sigma_{0}$, as a function of $N=\bar{m}_{\sigma} t / 2 \pi$, in the case $M \gg H$. Note that the number of oscillations of the field $\sigma$ is about $N / 3$, while the amplitude of oscillations of both fields is large. This figure corresponds to $\lambda=10^{-2}, g=1, m=10^{3} \mathrm{GeV}, M=1.3 \times 10^{11} \mathrm{GeV}$.

transfer all energy of the oscillating scalar field $\sigma$ to $\sigma$ particles (this is the leading process). The initial energy density of the field $\sigma$ is $M^{4} / 4 \lambda$. Each produced particle carries the energy $\sim \bar{m}_{\sigma} \sim \sqrt{2} M$. This implies that one should have $n_{k}$ $\sim \lambda^{-1}$ in order to transfer all energy of oscillations into $\sigma$ particles. The total duration of the process in terms of the number of oscillation $N$ follows from the relation $\exp (4 \pi \mu N) \sim \lambda^{-1}$, which gives $N \sim(4 \pi \mu)^{-1} \ln \lambda^{-1}$. For $\mu$ $\sim 0.3$ and $\lambda \sim 10^{-2}$ one has $N=O(1)$, i.e. the whole process finishes within one or two oscillations; the part of Figs. 6 and 7 at $N \gg 1$ is redundant.

The unusual efficiency of the process of $\sigma$ particle production can be explained by the relatively large value of the coupling constant $\lambda$ and by the tachyonic instability of the field at the first stages of its rolling down. A detailed theory of this process will be considered elsewhere [19]. A simple interpretation of our results is as follows.

The process of falling down of the field $\sigma$ in the regime $\lambda \ll g^{2}$ occurs almost independently of the field $\phi$. The field $\phi$ triggers the process, but then the field $\sigma$ moves essentially as a field falling down from the point $\sigma=0$ in the theory with the effective potential $\lambda /(4)\left(\sigma^{2}-\sigma_{0}\right)^{2}$. As we discussed in Sec. III, neglecting expansion of the universe, fluctuations of the field $\sigma$ in the beginning of the process grow as $\exp \sqrt{k^{2}-M^{2}} t$. Initially, there was no homogeneous classical field $\sigma$; the whole process of spontaneous symmetry breaking occurs due to the growth of quantum fluctuations. The value of the homogeneous mode $\langle\sigma\rangle$ vanishes all the time; spontaneous symmetry breaking occurs when $\sqrt{\left\langle\sigma^{2}\right\rangle}$ created by the instability approaches $\sigma_{0}$. As a result, already after the quarter of the first oscillation, $\sqrt{\left\langle\sigma^{2}\right\rangle}$ approaches $\sigma_{0}$, all energy will be concentrated not in a homogeneous field $\sigma$ but in its fluctuations with a wavelength $l$ $\sim 2 \pi M^{-1} \ln ^{1 / 2}\left(4 \pi^{2}\right) / \lambda$, which is somewhat greater than $M^{-1}$ [19]. In this sense one may even say that preheating ends within one quarter of an oscillation. This, however, would be not quite correct because after rolling there will be a stage of resonant production of particles with momenta comparable to $M$. 

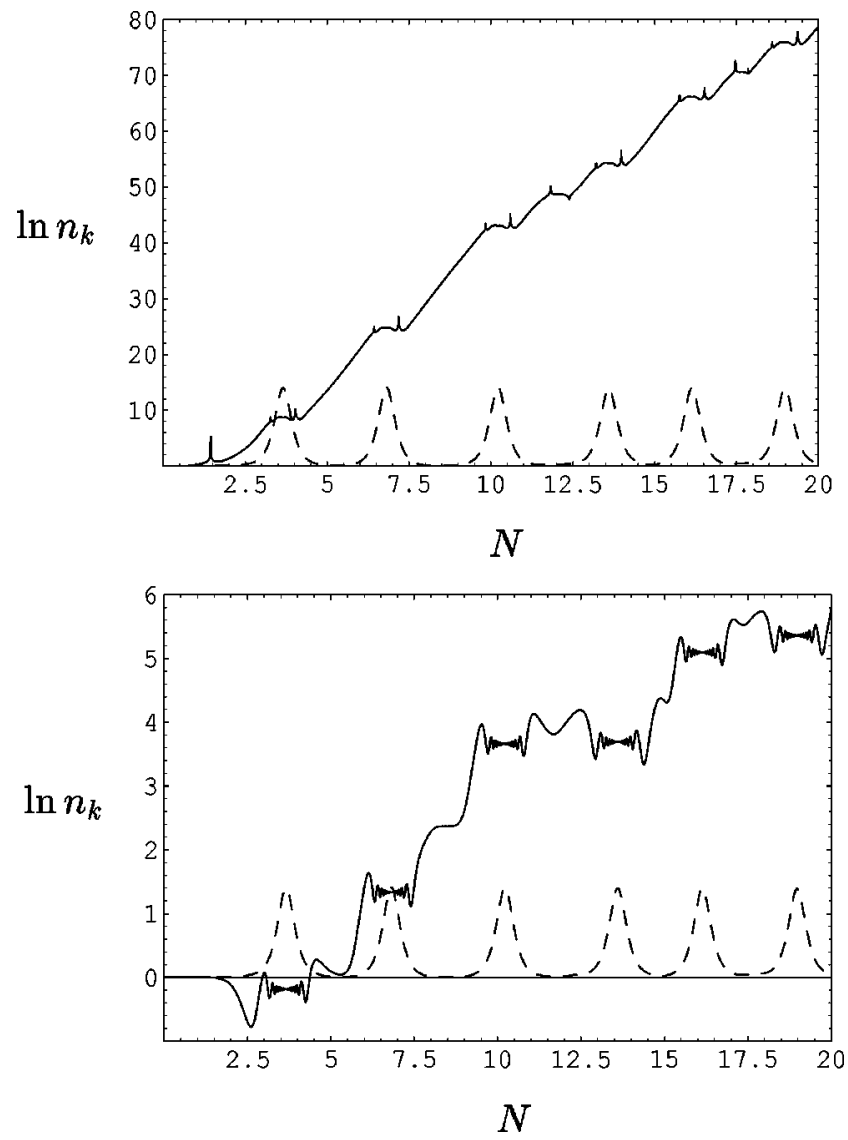

FIG. 6. The top panel shows the exponential growth of the occupation number $n_{k}$ of $\sigma$ particles, as a function of $N=\bar{m}_{\sigma} t / 2 \pi$, for $k=0.43 M$ and the parameters of Fig. 5. It acquires a typical growth parameter $\mu_{k} \simeq 0.3$ during the last stages of preheating. The lower panel shows the occupation number $n_{k}$ of $\phi$ particles, for $k$ $=0.2 M$. The growth parameter is an order of magnitude smaller, $\mu_{k} \simeq 0.023$, in this case. The dashed line shows the oscillations of the field $\sigma$. The upper panel shows $10 \sigma / \sigma_{0}$, while the lower panel shows $\sigma / \sigma_{0}$. The number of the $\sigma$ field oscillations differs from $N=\bar{m}_{\sigma} t / 2 \pi$ because the oscillations are not harmonic.

We should emphasize, however, that here again we deal not with the decay of the field $\sigma$ to other particles, but with a rapid process of transformation of the energy of the classical field $\sigma$ to the energy of $\sigma$ particles. The subsequent decay of these particles occurs in accordance with the elementary theory of reheating [2].

Let us now briefly discuss the case when the two couplings $g^{2}$ and $\lambda$ are of similar magnitude.

\section{Case $g^{2} \simeq \lambda$}

This case corresponds to the simplest supergravity hybrid inflation model $[17,20,21]$, based on a superpotential of the type

$$
W=S\left(\kappa \bar{\phi} \phi-\mu^{2}\right)
$$

which induces a scalar potential with self-coupling $\lambda=\kappa^{2}$ for the triggering field $\phi$, and couples the two fields with
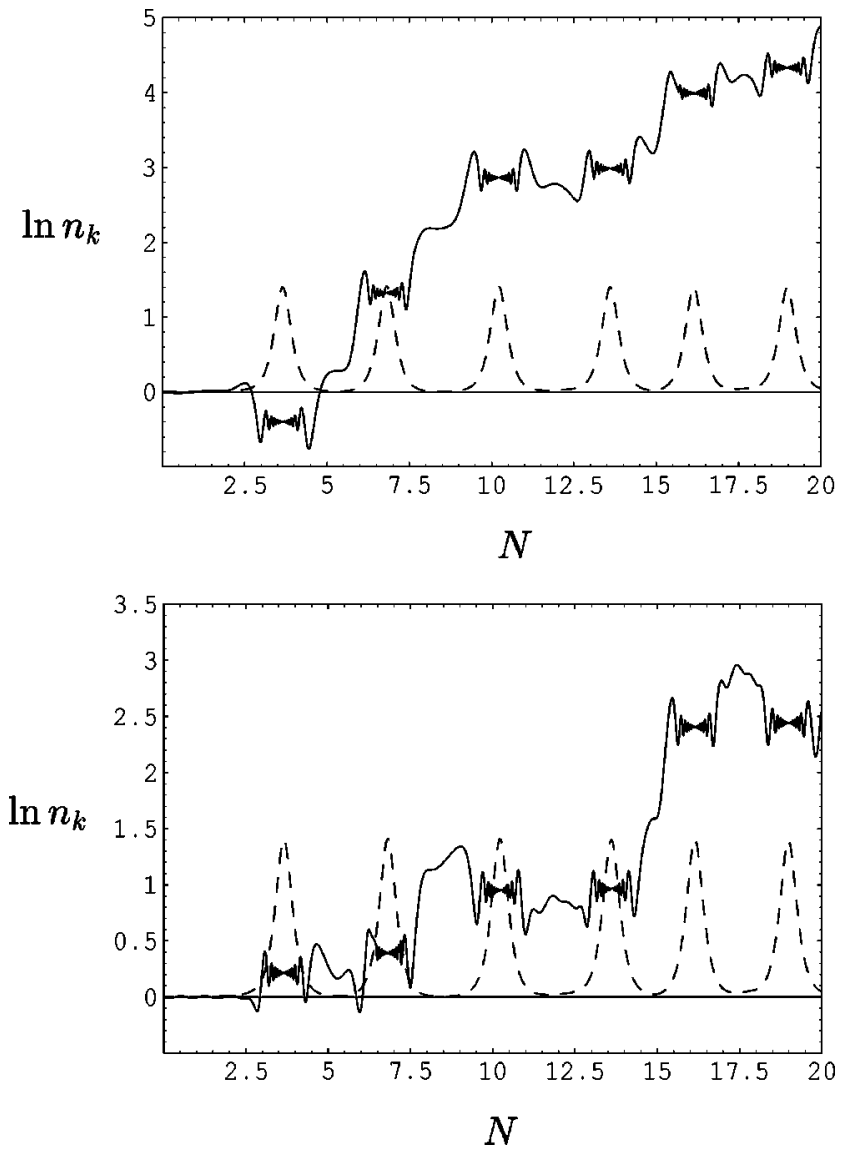

FIG. 7. The top panel shows the occupation number $n_{k}$ of $\chi$ particles as a function of $N=\bar{m}_{\sigma} t / 2 \pi$, for $k=0.2 M, h_{1}=0.3$, $h_{2}=1$ and the parameters of Fig. 5. It acquires a typical growth parameter $\mu \simeq 0.03$ during the last stages of preheating. The lower panel shows the same as above but for $h_{1}=h_{2}=1$. The growth parameter is somewhat smaller, $\mu \simeq 0.013$, in this case. The dashed line shows $\sigma / \sigma_{0}$.

strength $g^{2}=\kappa^{2}$, see Eq. (1). Thus, we have the same coupling, $\lambda=g^{2}$, as one should expect from supersymmetry universality. $^{2}$

In this case, the motion is always two-dimensional in the $(\sigma, \phi)$ field space: the field $\phi$ oscillates around zero while the field $\sigma$ oscillates around $\sigma_{0}$, with similar amplitudes but varying frequencies, see Fig. 8. Since the fields are coupled, their effective masses also vary. This induces the chaotic behavior of the fields shown in Fig. $8 .^{3}$ We have taken as parameters, $\quad \lambda=g^{2}=10^{-6}, \quad M=10^{-6} M_{\mathrm{P}}, \quad m=2$ $\times 10^{-10} M_{\mathrm{P}}$. The amplitude of the fields takes many oscillations to decrease significantly, e.g. after $N=50$, the amplitude of the $\phi$ field is still of order $1 / 2$, although after one $e$-fold (corresponding here to $N \simeq 340$ oscillations of the $\sigma$ field) it decays like $1 / t$ with amplitude $\Phi_{0} \sim 1 / 5$. Meanwhile its frequency is rather chaotic for the first $e$-fold, as one can

\footnotetext{
${ }^{2}$ This is not a general rule. For example, it is not necessarily true in D-term hybrid inflation [22], where the couplings $g^{2}$ and $\lambda$ come from the $\mathrm{F}$ and the $\mathrm{D}$ terms respectively.

${ }^{3}$ For a general discussion of chaos hybrid inflation and in preheating, respectively, see Refs. [23] and [7, 24].
} 

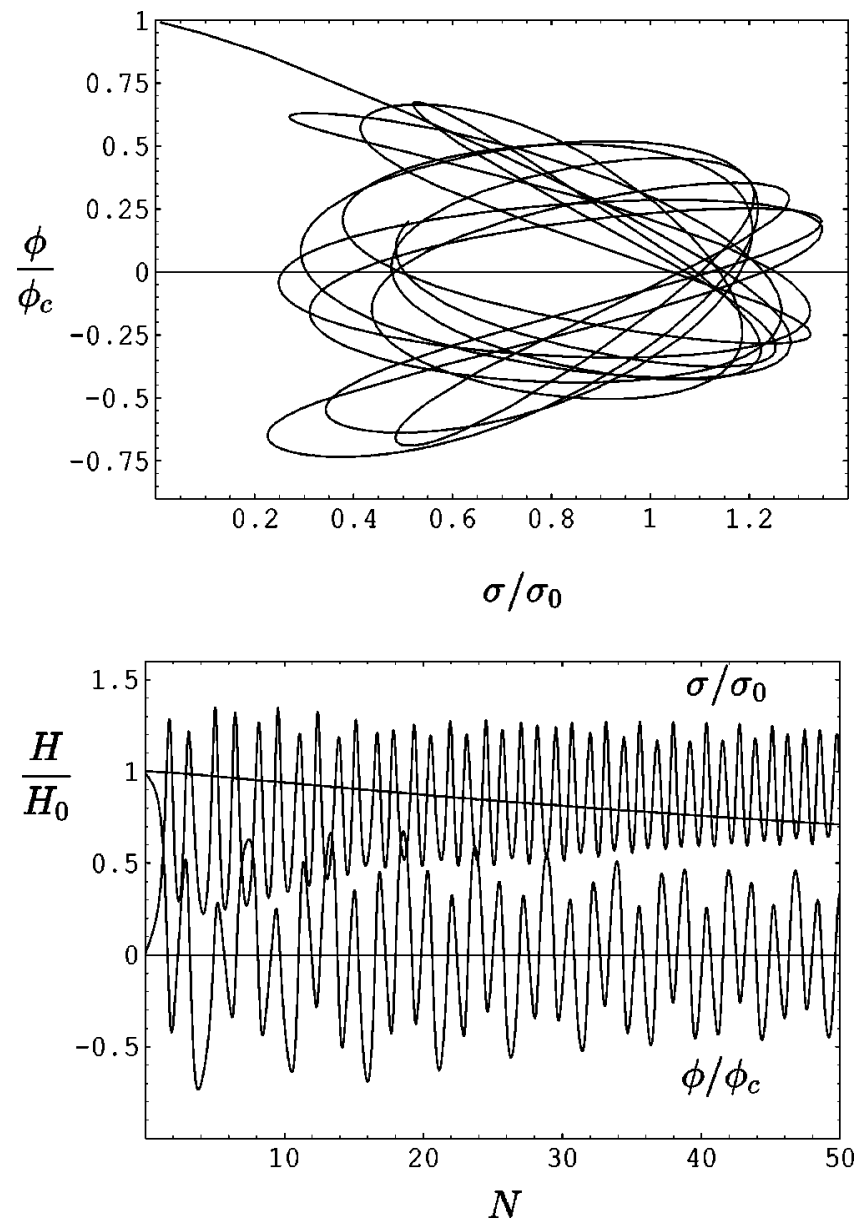

FIG. 8. The top panel shows the motion of the two scalar fields in the plane $\left(\sigma / \sigma_{0}, \phi / \phi_{c}\right)$. The lower panel shows the evolution after the end of inflation of $H / H_{0}, \phi / \phi_{c}$ and $\sigma / \sigma_{0}$, as a function of the number of oscillations of the field $\sigma, N=\bar{m}_{\sigma} t / 2 \pi$. It is clear that for the first dozens of oscillations, the behavior of both fields is extremely chaotic, and the amplitude of the fields decreases rather slowly compared to previous cases. These figures correspond to the parameters $\lambda=g^{2}=10^{-6}, M=10^{-6} M_{\mathrm{P}}, m=2 \times 10^{-10} M_{\mathrm{P}}$.

appreciate in Fig. 8, but eventually regularizes and becomes oscillatory, while the rate of expansion approaches the usual dust dominated behavior, $\epsilon=-\dot{H} / H^{2}=3 / 2$. This can be appreciated in Fig. 9.

Even though initially the chaotic behavior of the fields' oscillations prevents any particle production, as soon as their behavior becomes regular (after about $1 e$-fold) the amplitude of the oscillations is large enough (thanks to the fact that it has not decreased significantly during the chaotic motion) that one may have some parametric resonance.

Like in the previous case $\left(g^{2} \gg \lambda\right)$, a new field $\chi$ could couple to $\sigma$ and $\phi$ with potential (15). Since $\sigma$ acquires the vacuum expectation value $\sigma_{0}$, we have to suppress the coupling to $\sigma$ in order to induce parametric resonance of $\chi$ particles. In this case, since $g^{2}=\lambda$, the condition (33) becomes $h_{1} \gg h_{2}$. Eventually the amplitude of the field $\phi$ oscillations will decrease to $\Phi(t)<h_{2} / h_{1}$ and the explosive production will end. The Mathieu equation is the same as in the previous case, see Eq. (35), with occupation number (38) and frequency (39).

We have taken in this case $h_{2} \simeq 0$ and $h_{1}=4 \times 10^{-3}$, and

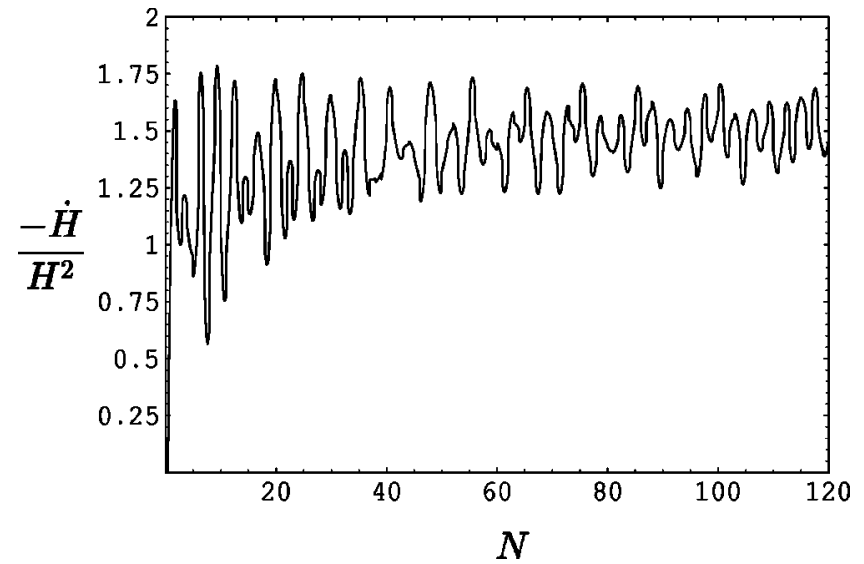

FIG. 9. The evolution of the parameter $\epsilon=-\dot{H} / H^{2}$ after the phase transition in the case $g^{2}=\lambda$. The dust dominated era, $\epsilon$ $=3 / 2$, is approached rather chaotically after many field oscillations, although in less than one $e$-fold.

found explosive production of $\chi$-particles, see Fig. 10. Note the initial plateau, as the chaotic behavior of $\phi$ prevents a significant production of $\chi$-particles. Eventually, the stochastic resonance regime begins, but the growth index $\mu_{k}$ $\simeq 0.03$ associated with the mode $k=M$ at the last stages of reheating is not as large as in the previous cases.

Back reaction of the produced $\chi$-particles on the oscillations of the $\phi$-field will become important at time $t_{1}$, given by Eq. (40), when the expectation value of the $\chi$-field becomes

$$
\left\langle\chi^{2}\right\rangle_{1} \simeq \frac{\bar{m}_{\phi}^{2}}{h_{1}^{2}}=\frac{M^{2}}{h_{1}^{2}},
$$

and the field $\phi$ acquires a large mass $\sim h_{1}^{2}\left\langle\chi^{2}\right\rangle_{1}$. Particle production still continues until the effective $q$ parameter enters the narrow resonance regime, $q \lesssim 1 / 4$ [7].

Symmetry restoration, if it exists, depends only on the couplings of $\chi$ to $\phi$ and $\sigma$, see Eq. (46),

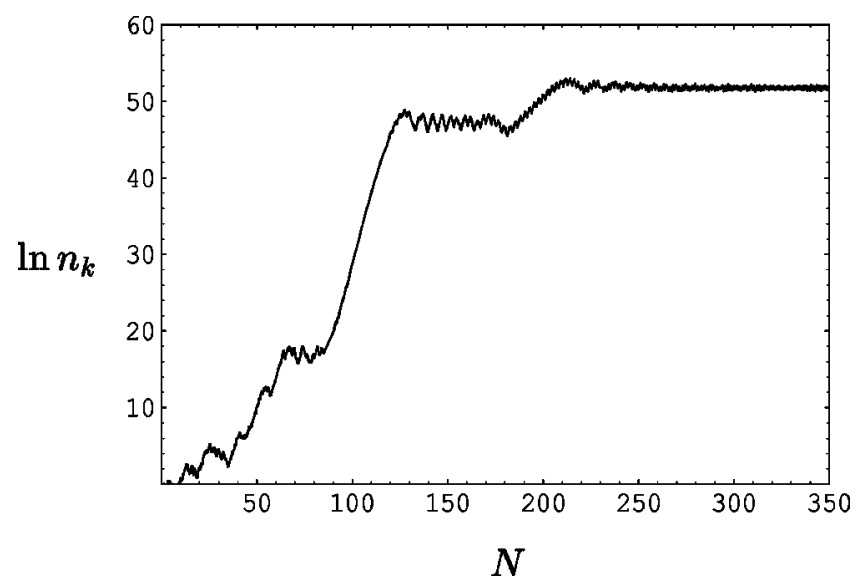

FIG. 10. The exponential growth of the occupation number $n_{k}$ of $\chi$-particles as a function of the number of oscillations of the field $\sigma, N=\bar{m}_{\sigma} t / 2 \pi$, for $k=M$ and the parameters of Fig. 8. One can distinguish here the chaotic plateau followed by the broad stochastic resonance regime. It acquires a typical growth parameter $\mu$ $\simeq 0.03$ during the last stages of preheating. 


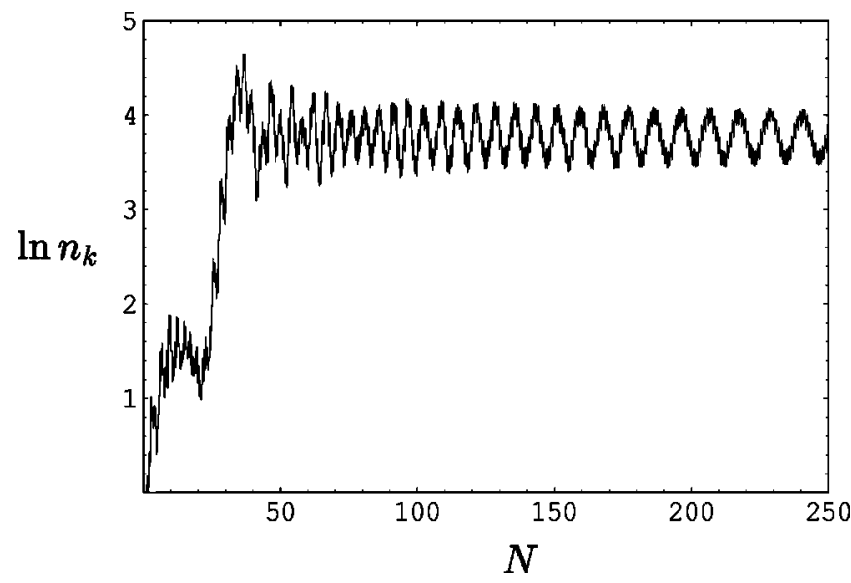

FIG. 11. The exponential growth of the occupation number $n_{k}$ of $\sigma$-particles as a function of the number of oscillations of the field $\sigma, N=\bar{m}_{\sigma} t / 2 \pi$, for $k=M$ and the parameters of Fig. 8. One can only distinguish here the narrow resonance regime. It acquires a typical growth parameter $\mu \simeq 0.01$ during the last stages of preheating.

$$
q_{1}^{1 / 2}>\frac{h_{1}^{2}}{4 h_{2}^{2}} \gg 1 .
$$

Although difficult, this condition could still be satisfied.

In this special case, there is also a small production of $\sigma$-particles, due to the oscillations of the homogeneous $\sigma$ and $\phi$-fields. The effective mass (20) of the $\sigma$-fluctuations in this case is

$$
\begin{aligned}
m_{\sigma}^{2}= & \bar{m}_{\sigma}^{2}+g^{2} \phi^{2}+3 \lambda\left(\sigma^{2}-\sigma_{0}^{2}\right), \\
= & \bar{m}_{\sigma}^{2}+M^{2} \Phi^{2} \sin ^{2} \bar{m}_{\phi} t \\
& +3 M^{2}\left(2 \Sigma \sin \bar{m}_{\sigma} t+\Sigma^{2} \sin ^{2} \bar{m}_{\sigma} t\right),
\end{aligned}
$$

where we have used Eqs. (24) and (47).

Since $\bar{m}_{\phi}^{2}=g^{2} M^{2} / \lambda \simeq M^{2}$ and $\bar{m}_{\sigma}^{2}=2 M^{2}$, the effective mass (63) oscillates with various frequencies. In this case, we cannot neglect the terms proportional to $\Phi^{2}$ and $\Sigma^{2}$, since they are not small and represent the oscillations of the two background fields. Initially, the motion is chaotic and no parametric resonance is possible. Eventually, oscillations become more regular, when the amplitude of oscillations is still large, $\Sigma \sim 1 / 2$ and $\Phi \sim 1 / 3$. Then the Mathieu equation for the production of $\sigma$ particles becomes

$$
\psi_{k}^{\prime \prime}+\left[A_{\sigma}(k)+2 q_{\sigma} \sin 2 z\right] \psi_{k}=0,
$$

where $z=\bar{m}_{\sigma} t / 2$ and

$$
\begin{aligned}
A_{\sigma}(k) & =\frac{4 k^{2}}{a^{2} \bar{m}_{\sigma}^{2}}+4, \\
q_{\sigma} & =\frac{12 M^{2}}{\bar{m}_{\sigma}^{2}} \Sigma .
\end{aligned}
$$

Initially we can neglect back reaction, and $\bar{m}_{\sigma}^{2}=2 M^{2}$. In this case $q_{\sigma}=6 \Sigma_{0} \simeq 3$, see Fig. $8 \mathrm{~b}$. There will be a slight produc- tion of $\sigma$-particles, as can be seen in Fig. 11. However, the effective growth parameter $\mu_{k} \simeq 0.01$ is never large enough to dominate the decay of the $\sigma$-field. Back reaction will be dominated by the $\chi$-particle production, certainly not by $\sigma$ particle production.

The existence of the regime of chaotic oscillations may be important in application to realistic models of hybrid inflation. For example, recently it was argued that in hybrid inflation scenario based on the $S U(5)$ model, spontaneous symmetry breaking has a tendency to occur in the direction with a "wrong", type of symmetry breaking $S U(4)$ $\times U(1)$, which was considered as a problem of this scenario [25]. However, an analogous result is true even for the standard high temperature phase transitions in the $S U(5)$ [26], where the phase transition is typically first order, and the bubbles of the phase $S U(4) \times U(1)$ form much more often than the bubbles of the phase $S U(3) \times S U(2) \times U(1)$ [27]. Still, the universe may eventually end up in the proper vacuum state $S U(3) \times S U(2) \times U(1)$, after the bubbles of the phase $S U(3) \times S U(2) \times U(1)$ "eat" the bubbles of all other phases $[27,1]$. Similarly, the phase transition in the $S U(5)$ version of the hybrid inflation model may be first order, with several different phases being produced. Then one should study the equilibrium between these phases. But even if the phase transition is second order, as in the simplest model considered above, one may wonder whether the chaotic behavior of the scalar fields $\phi$ and $\sigma$ may split the universe into many domains with different types of symmetry breaking. Then one should study which of these domains will eventually survive. This process becomes even more complicated if one studies back reaction of $\chi$ particles produced during preheating on the shape of the effective potential. The best way to investigate this issue is to use lattice simulations $[12,19]$.

\section{REHEATING IN HYBRID MODELS WITH TWO STAGES OF INFLATION}

So far we have studied the generic behavior of preheating in various hybrid inflation models. We see that in some of them it is indeed possible to produce large amounts of particles in an explosive way and thus reheat the universe rather efficiently. In all models which we studied so far inflation ends at the moment when the field $\phi$ reaches its critical value $\phi_{c}$ and a phase transition with the generation of the classical field $\sigma$ occurs.

However, this is not the most general situation. There are some hybrid inflation models where inflation ends before the phase transition, see e.g. Refs. [20,21]. There is also an interesting class of models where inflation continues for a while after the phase transition $[13,14]$. This happens if the symmetry breaking field has a vacuum expectation value $\sigma_{0} \sim M_{\mathrm{P}}$, and a mass of order $M \sim 1 \mathrm{TeV}$. This requires an extremely small parameter $\lambda \ll g^{2}$. As a consequence, after the phase transition at $\phi=\phi_{c}$, the symmetry breaking field slowly rolls down its potential while still inflating the universe, until it starts oscillating around $\sigma_{0}$, ending inflation. The amplitude of oscillations is very small compared with $\sigma_{0}$, i.e. $\Sigma \ll 1$.

As we already discussed in Sec. IV B, in this case preheating is inefficient unless there exists a field $\chi$ strongly 
coupled to $\sigma$, which, however, does not acquire mass greater than $M$ after spontaneous symmetry breaking. It is especially difficult to arrange it in the case under consideration, because the amplitude of spontaneous symmetry breaking is $\sim M_{\mathrm{P}}$, which is 16 orders of magnitude greater than $M$. Thus one may expect that even a very weak interaction of the field $\sigma$ with $\chi$ will make the field $\chi$ too heavy. In Sec. IV B we mentioned that it is possible to have light particles which are strongly coupled to the field $\sigma$, but it requires either fine tuning or introduction of flat directions. We will not discuss this possibility here.

Now let us consider a possibility of a perturbative decay of the oscillations of the field $\sigma$. In general, this process could be efficient if this field has a renormalizable interaction with some light fields, with a large coupling constant $\alpha^{2}$. However, there are two problems associated with this possibility. First of all, as we just mentioned, such an interaction typically makes the fields interacting with the field $\sigma$ extremely heavy, with a mass $\sim \alpha \sigma_{0} \sim \alpha M_{\mathrm{P}}$. Such particles cannot be produced by perturbative decay of the particles with mass $M \sim 1 \mathrm{TeV}$ unless $\alpha$ is extremely small, $\alpha$ $<10^{-16}$. But in this case the decay rate is extremely small too.

However, even if one finds a way to have some light particles strongly coupled to the field $\sigma$, its decay still occurs very slowly. Indeed, suppose that the decay rate $\Gamma$ is very large, $\Gamma \sim M$. Then naively one could expect that the field decays within the time $\Delta t \sim \Gamma^{-1} \sim M^{-1}$, which is of the same order of magnitude as the Hubble constant at the end of inflation in this model. In such a case the energy density of the oscillating scalar field $\rho_{\sigma} \sim M^{2} \sigma_{0}^{2} \sim M^{2} M_{\mathrm{P}}^{2}$ would be rapidly converted to the thermal energy $\sim T^{4}$, which would heat the universe up to the temperature $T_{r} \sim 10^{-1} \sqrt{M M_{\mathrm{P}}} \sim 10^{7} \mathrm{M}$ [7]. However, this is hardly possible. Indeed, strongly interacting particles in thermal equilibrium typically acquire effective mass $\sim T$. As soon as this mass becomes comparable to $M$, reheating shuts down.

As a result, instead of rapidly reaching the temperature $T_{r} \sim 10^{-1} \sqrt{M M_{\mathrm{P}}}$, the universe remains at the nearly constant temperature $T \sim M \sim 1 \mathrm{TeV}$. The decay of the field $\sigma$ continues for a very long time, until the energy density of this field drops down from $M^{2} M_{\mathrm{P}}^{2}$ to $M^{4}$; see Ref. [28] for a discussion of a similar effect. Thus, reheating temperature in this model may be very small; reheating may end just before the electroweak phase transition or even after it. This may have important implications for the theory of baryogenesis and for the cosmological moduli problem.

But the situation may be even more interesting and complicated. Indeed, as was noted in Ref. [13], the existence of a second stage of inflation in this model may produce a very narrow peak in the spectrum of density perturbations. A further investigation of this issue have shown that this peak is in fact much higher than originally expected, see Ref. [14]. The density perturbations corresponding to this peak may lead to a copious black hole production even if the universe after inflation rapidly enters the radiation dominated stage. However, this effect becomes even much more pronounced if the universe for a long time remains matter dominated, because without radiation pressure there is nothing to prevent gravitational collapse. Therefore even if the peak in spectrum of density perturbations is not very high, it may still lead to a copious black hole formation.

If our analysis of reheating in this model is correct, then reheating completes at a time which is at least 16 orders of magnitude longer than the age of the universe at the end of inflation. All this time the universe remains matter dominated. In this case we expect a huge number of black holes with a narrow range of masses to be produced soon after inflation, so that most of the matter after inflation will be in the form of primordial black holes. If the second stage of inflation is prolonged, the masses of the black holes will be extremely large, which will lead to unacceptable cosmological consequences. However, for a wide range of the parameters the second stage of inflation will be relatively short. In such a case the black hole masses will be very small, and they will evaporate before primordial nucleosynthesis. The peak in the spectrum of density perturbations in this model is very narrow. As a result, all black holes will have mass of the same order of magnitude. Evaporation of these black holes will happen almost simultaneously, at the time related to their mass. This process will produce all the radiation and matter we see in the universe today [14].

\section{CONCLUSIONS}

In this paper we considered the initial stages of reheating in the hybrid inflation scenario. We have found that in certain cases this process begins with a stage of parametric resonance. In such a situation the standard perturbative approach to the theory of reheating should be considerably modified. But even in the situations where preheating is inefficient, a detailed investigation of the behavior of the coupled system of two fields $\phi$ and $\sigma$ is necessary for a proper investigation of the perturbative reheating regime. For example, as we have shown, for $\lambda \gg g^{2}$ all energy after inflation is stored in the oscillations of the field $\phi$, so one should study perturbative or nonperturbative decay of this field. For $\lambda \ll g^{2}$ all energy is stored in the oscillations of the field $\sigma$, so one should study its decay. Meanwhile for $\lambda \sim g^{2}$ the fields enter a regime of chaotic oscillations where energy density is transferred back and force between the fields $\phi$ and $\sigma$. Investigation of this regime may be interesting not only for the investigation of reheating, but also for the study of symmetry breaking pattern in realistic models of hybrid inflation. We have found that preheating can be very efficient if the effective masses of the fields $\phi$ and $\sigma$ are much greater than the Hubble constant at the end of inflation, or if these fields are coupled to other light scalar (or vector) fields $\chi$.

In addition to the simplest hybrid inflation scenario [9], we also studied a recently proposed scenario with two stages of inflation $[13,14]$. Rather unexpectedly, we found that preheating, as well as the standard mechanism of reheating, in this scenario is extremely inefficient. In such a situation, reheating may occur in a very unusual way, via formation of primordial black holes and their subsequent evaporation. Even though this process may seem somewhat exotic, it may be the leading mechanism of reheating in inflationary models with a peak in the spectrum of density perturbations [14], as well as in a much more general class of hybrid inflationary models with a blue spectrum of density perturbations. 


\section{ACKNOWLEDGMENTS}

J.G.B. thanks G. Dvali and J. R. Espinosa for useful discussions. He is also grateful to the Physics Department at Stanford University, for their hospitality during part of this work. A.L. is very grateful to L. Kofman and I. Tkachev for the discussions of the theory of preheating. The work of A.L. was supported by NSF grants PHY-9219345 and AST9529225. This work was also supported by a NATO Collaborative Research Grant, Ref. CRG.950760.
[1] A. D. Linde, Particle Physics and Inflationary Cosmology (Harwood, Chur, Switzerland, 1990).

[2] A. D. Dolgov and A. D. Linde, Phys. Lett. 116B, 329 (1982); L. F. Abbott, E. Fahri, and M. Wise, ibid. 117B, 29 (1982).

[3] L. Kofman, A. D. Linde, and A. A. Starobinsky, Phys. Rev. Lett. 73, 3195 (1994).

[4] J. Traschen and R. Brandenberger, Phys. Rev. D 42, 2491 (1990); Y. Shtanov, J. Traschen, and R. Brandenberger, ibid. 51, 5438 (1995); D. Boyanovsky, H. J. de Vega, R. Holman, D. S. Lee, and A. Singh, ibid. 51, 4419 (1995).

[5] L. A. Kofman, A. D. Linde, and A. A. Starobinsky, Phys. Rev. Lett. 76, 1011 (1996); I. Tkachev, Phys. Lett. B 376, 35 (1996).

[6] E. W. Kolb, A. D. Linde, and A. Riotto, Phys. Rev. Lett. 77, 4290 (1996); G. W. Anderson, A. D. Linde, and A. Riotto, ibid. 77, 3716 (1996).

[7] L. Kofman, A. D. Linde, and A. A. Starobinsky, Phys. Rev. D 56, 3258 (1997).

[8] P. B. Green, L. Kofman, A. D. Linde, and A. A. Starobinsky, Phys. Rev. D 56, 6175 (1997).

[9] A. D. Linde, Phys. Lett. B 259, 38 (1991); Phys. Rev. D 49, 748 (1994).

[10] J. García-Bellido and A. D. Linde, Phys. Lett. B 398, 18 (1997); Phys. Rev. D 55, 7480 (1997).

[11] G. Lazarides, R. K. Schaefer, and Q. Shafi, Phys. Rev. D 56, 1324 (1997); G. Dvali, G. Lazarides, and Q. Shafi, hep-ph/9710314, 1997.

[12] S. Yu. Khlebnikov and I. I. Tkachev, Phys. Rev. Lett. 77, 219 (1996); T. Prokopec and T. G. Roos, Phys. Rev. D 55, 3768 (1997); S. Khlebnikov and I. Tkachev, Phys. Rev. Lett. 79,
1607 (1997); S. Khlebnikov and I. Tkachev, Phys. Rev. D 56, 653 (1997); B. R. Greene, T. Prokopec, and T. G. Roos, ibid. 56, 6484 (1997).

[13] L. Randall, M. Soljačić, and A. H. Guth, Nucl. Phys. B472, 377 (1996).

[14] J. García-Bellido, A. D. Linde and D. Wands, Phys. Rev. D 54, 6040 (1996).

[15] A. R. Liddle and D. H. Lyth, Phys. Rep. 231, 1 (1993).

[16] J. García-Bellido and D. Wands, Phys. Rev. D 54, 7181 (1996).

[17] E. J. Copeland, A. R. Liddle, D. H. Lyth, E. D. Stewart, and D. Wands, Phys. Rev. D 49, 6410 (1994).

[18] C. Lineweaver and D. Barbosa, astro-ph/9706077, 1997.

[19] I. Tkachev, L. A. Kofman, A. D. Linde, A. Starobinsky, and S. Yu. Khlebnikov (in preparation).

[20] G. Dvali, Q. Shafi, and R. K. Schaefer, Phys. Rev. Lett. 73, 1886 (1994).

[21] A. D. Linde and A. Riotto, Phys. Rev. D 56, 1841 (1997).

[22] P. Binetruy and G. Dvali, Phys. Lett. B 388, 241 (1996); E. Halyo, ibid. 387, 43 (1996); D. H. Lyth and A. Riotto, ibid. 412, 28 (1997).

[23] N. J. Cornish and J. J. Levin, Phys. Rev. D 53, 3022 (1996); R. Easther and K. Maeda, gr-qc/9711035, 1997.

[24] B. A. Bassett, hep-ph/9709443, 1997.

[25] G. Dvali, L. M. Krauss, and H. Liu, hep-ph/9707456, 1997.

[26] V. A. Kuzmin, M. E. Shaposhnikov, and I. I. Tkachev, Z. Phys. C 12, 83 (1982).

[27] A. D. Linde, "Kinetics of Phase Transitions in Grand Unified Theories,' Lebedev Institute Preprint No. 266, 1981.

[28] A. D. Linde, Phys. Lett. 160B, 243 (1985). 